\title{
Características de las agresiones sexuales en beneficiarias del programa de tratamiento de depresión de un consultorio de atención primaria
}

\author{
FRANCISCO IBACETA $^{(1)}$, CAROLINA GUADALUPE( ${ }^{(1)}$, KARINA GONZÁLEZ $^{(2)}$, ADA ZAMBRANO $^{(3)}$ y $^{2}$ \\ CAROLINA BECAR ${ }^{(4)}$
}

\section{RESUMEN}

Las agresiones sexuales sufridas en la infancia son factores de riesgo psicológico para el desarrollo de sicopatologías en la adultez. El objetivo de este estudio fue conocer la prevalencia y características de las agresiones sexuales sufridas en la infancia por las beneficiarias del programa de depresión del consultorio de atención primaria de la comuna de Vitacura en Santiago de Chile. Se entrevistó a 29 pacientes beneficiarias del programa de depresión a través de una pauta de recolección de información sobre las características de las agresiones sexuales sufridas en la infancia. A todas también se les aplicó la escala de depresión de Hamilton. La prevalencia de agresiones sexuales en la infancia se encuentra en el rango establecido por investigaciones restropectivas más amplias. Esto es cercana al $25 \%$ en población adulta femenina que está cursando un episodio depresivo. Destaca el alto nivel de traumatización de la muestra estudiada, lo que indica una tendencia hacia una vulnerabilidad neurobiológica al desarrollo de cuadros de morbilidad en salud mental en la adultez.

Palabras clave: depresión, agresión sexual, psicoterapia.

\section{ABSTRACT}

CHARACTERISTICS OF SEXUAL AGGRESSIONS AMONG WOMEN BENEFITING FROM THE DEPRESSION THERAPY PROGRAM OF A PRIMARY CARE OUTPATIENT CLINIC

Sexual aggressions suffered during childhood are a psychological risk factor for the development of psychopathologies during adulthood. The purpose of the present study was to assess the prevalence and characteristics of sexual aggressions suffered during childhood by women beneficiary of the depression therapy program from the primary care outpatient clinic of the Vitacura commune in Santiago, Chile. Twenty-nine (29) program beneficiary women were interviewed following a data collection guideline that assessed the characteristics of sexual aggressions suffered during childhood. The Hamilton rating scale for depression was applied to all individuals. The prevalence of sexual aggressions during childhood among female adults undergoing an episode of depression was close to $25 \%$, a figure that is within the range established by larger scale retrospective research. The high degree of trauma among the individuals of the study sample was striking, thus indicating

(1) Centro de Salud Familiar Vitacura. Indiana 1195 Vitacura. Santiago. Chile. ibaceta@yahoo.com

(2) Consejo de Defensa del Niño. Castro. Chile.

(3) Programa Previene Temuco. Chile.

(4) Consejo de Defensa del Niño. Quilicura. Chile. 
a tendency towards a neurobiological vulnerability to develop mental health morbid conditions in adulthood.

Keywords: depression, sexual aggression, psychotherapy.

\section{INTRODUCCIÓN}

Se ha establecido que el estrés en etapas tempranas del desarrollo, en conjunción con predisposiciones biológicas, contribuyen a generar una vulnerabilidad de largo plazo al desarrollo de morbilidad de salud mental ${ }^{1}$. En particular, las agresiones sexuales sufridas en la infancia, en tanto trauma, son consideradas factores de riesgo psicológico para el desarrollo de psicopatología en la adultez. Se sostiene que a mayor gravedad y cronicidad de las agresiones sexuales sufridas, mayor puede ser la gravedad de las manifestaciones psicopatológicas en la vida adulta ${ }^{2}$.

Si bien no es posible establecer que la agresión sexual infantil sea un factor etiológico único y específico en el desarrollo de trastornos psiquiátricos en la adultez, se ha reportado que existiría una relación manifiesta entre ésta y el desarrollo de episodios depresivos en la vida adulta $^{3}$. En particular, se ha sugerido que existe un período crítico en el desarrollo del ser humano en el que la experiencia de trauma puede conllevar cambios neurobiológicos permanentes, que aumentan la posibilidad de desarrollar trastornos del estado del ánimo como respuesta a la experiencia de otros estresores posteriores en la vida de la persona ${ }^{4}$.

La depresión es una patología frecuente, con elevados índices de discapacidad y que genera significativos costos económicos ${ }^{5}$. En el sistema de atención primaria chileno se ha estimado una prevalencia cercana al $30 \%$.

Para enfrentar este problema, desde mediados del año 2001 el Ministerio de Salud se encuentra ejecutando el Programa Nacional de Detección Diagnóstico y Tratamiento Integral de la Depresión en Atención Primaria.

En la guía clínica de este programa ${ }^{6}$ se describen factores de riesgo psicosocial que, como con- diciones pasadas o presentes, pueden determinar el inicio, la evolución y el pronóstico del cuadro depresivo. Sin embargo, a pesar de la fuerte evidencia que relaciona a la agresión sexual en la infancia con el desarrollo de trastorno depresivos en la vida adulta, no se menciona este evento traumático como factor de riesgo potencial a considerar al momento de realizar la evaluación de los posibles beneficiarios del programa.

Consideramos que si esta condición puede ser frecuente entre las personas que ingresan al programa y si adicionalmente, tal como se ha expuesto, se relaciona con la presentación del cuadro depresivo, sin duda alguna se la debe considerar al momento de realizar un acercamiento integral y ciertamente efectivo.

El propósito de este trabajo fue establecer la prevalencia y características de las agresiones sexuales en la infancia sufridas por las mujeres beneficiarias del programa de depresión del consultorio de atención primaria de la comuna de Vitacura en Santiago de Chile.

\section{MATERIAL Y MÉTODO}

La investigación se realizó en el Centro de Salud Familiar (CESFAM) de la comuna de Vitacura. Entre los meses de abril a septiembre del año 2006. Durante la entrevista de evaluación para el ingreso al programa de depresión se preguntó a todas los pacientes si habían sido víctimas de agresiones sexuales antes de los 16 años. A quienes afirmaron haber sido víctimas de agresiones sexuales se le solicitó autorización para ser entrevistadas a través de una pauta de recolección de información, construida específicamente para desarrollar esta investigación, sobre las características de las agresiones sexuales sufridas. Ninguna rechazó ser parte del estudio.

Por otra parte, a todas las personas que par- 
ticiparon de este estudio se les aplicó la Escala de Depresión de Hamilton. Ésta es una escala de evaluación externa, en donde la administración es realizada por un observador quien es el que cuantifica la intensidad de la depresión.

En términos generales, se consultó a las entrevistadas en relación a los siguientes tópicos relacionados con las agresiones sexuales:

- Edad en que ocurrieron las agresiones sexuales.

- Duración (como parte del desarrollo vital) y frecuencia de las mismas.

- Tipo de agresión sexual.

- Vínculo con el agresor.

- Si ocurrió revelación de las agresiones.

- Situaciones que pueden agravar el impacto traumático.

La información obtenida se organizó en tablas de frecuencia de acuerdo a cada una de las variables exploradas.

\section{RESULTADOS}

Durante el período de abril a septiembre del año 2006 ingresaron 107 pacientes, todas de sexo femenino, al programa de depresión. 29 de ellas (27.1\%) afirmó haber sufrido agresiones sexuales antes de los 16 años. La edad promedio de este grupo de 29 pacientes fue de 41.5 años, siendo el rango de edad entre los 20 y 56 años. Al momento de la entrevista para esta investigación un $36 \%$ estaba soltera, un $24 \%$ separada, un $20 \%$ mantenía una relación de convivencia, mientras que un $17 \%$ estaba casada.

En relación a las características de las agresiones sexuales sufridas (Tabla 1), el $90 \%$ de las agresiones sexuales ocurre antes de los 13 años. Alrededor de un $82 \%$ de los casos se trataría de agresiones sexuales crónicas, es decir, de una duración mayor a un año. En relación con la frecuencia ésta se presentó mayor a diez episodios en casi $76 \%$ de la muestra.
Tabla 1. Características de las agresiones sexuales

\begin{tabular}{|l|c|}
\hline EDAD & N CASOS \\
\hline $0-6$ & $13(45 \%)$ \\
\hline $7-12$ & $13(45 \%)$ \\
\hline 13 y más & $3(10 \%)$ \\
\hline DURACIÓN & $5(17 \%)$ \\
\hline Menor a 1 año & $13(45 \%)$ \\
\hline $1-5$ años & $11(38 \%)$ \\
\hline Mayor a 6 años & $1(3.5 \%)$ \\
\hline FRECUENCIA EPISÓDICA & $6(20.7 \%)$ \\
\hline Episodio único & $22(75.9 \%)$ \\
\hline $2-9$ & \\
\hline Más de 10 & \\
\hline
\end{tabular}

Respecto al vínculo con el agresor (Tabla 2), la mayor frecuencia se encuentra en agresiones sexuales por parte de miembros de la familia, siendo la de mayor prevalencia dentro de este grupo la de carácter incestuoso en un 55.1\% del total de las mujeres estudiadas.

Tabla 2. Vínculo con el agresor

\begin{tabular}{|c|c|c|c|}
\hline \multirow{2}{*}{ Intra-familiar } & $\begin{array}{c}\text { Incestuoso } \\
\text { (Padre o } \\
\text { Padrastro) }\end{array}$ & $\begin{array}{c}\text { Otro } \\
\text { Familiar }\end{array}$ & Total \\
\cline { 2 - 4 } & $\begin{array}{c}16 \\
(55.1 \%)\end{array}$ & $\begin{array}{c}8 \\
(27.6 \%)\end{array}$ & $\begin{array}{c}24 \\
(83 \%)\end{array}$ \\
\hline Extrafamiliar & \multicolumn{3}{|c|}{$5(17 \%)$} \\
\hline
\end{tabular}

En lo referido a las características de las agresiones sexuales (Tabla 3), un 55.2\% de mujeres consultantes señaló haber sufrido violación, un $37.9 \%$ abuso sexual y un $27.6 \%$ abuso sexual sin contacto físico. Cabe destacar aquí que las frecuencias son compuestas. Es decir, prácticamente la totalidad de las mujeres que fueron violadas refirieron un período anterior de abuso sexual, ya sea por parte del mismo agresor o de otra persona. Sólo las personas que fueron víctimas de 
agresiones sexuales sin contacto físico refirieron un agresor y un solo tipo de agresión.

Tabla 3. Tipo de agresión sexual

\begin{tabular}{|l|c|}
\hline Sin contacto físico & $8(27.6 \%)$ \\
\hline $\begin{array}{l}\text { Abuso sexual (contacto físico } \\
\text { sin penetración) }\end{array}$ & $11(37.9 \%)$ \\
\hline Violación & $16(55.2 \%)$ \\
\hline
\end{tabular}

En relación a la develación de las agresiones sexuales (Tabla 4), un 58.6\% de las entrevistadas declaró no haber revelado las agresiones sexuales, mientras que el $41.4 \%$ restante señala haber develado las agresiones a algún miembro significativo de su familia. El 90\% que dijo haber revelado sostiene que la respuesta fue de ausencia de protección. Ninguno de esos casos fue denunciado ante instancias policiales y/o judiciales.

Tabla 4. Revelación de agresión sexual

\begin{tabular}{|l|l|l|c|}
\hline \multirow{2}{*}{ Revelación } & Protección & $\begin{array}{l}\text { No } \\
\text { Protección }\end{array}$ & Total \\
\cline { 2 - 4 } & $2(6.9 \%)$ & $10(34.5 \%)$ & $12(41.4 \%)$ \\
\hline $\begin{array}{l}\text { No } \\
\text { revelación }\end{array}$ & \multicolumn{3}{|c|}{$17(58.6 \%)$} \\
\hline
\end{tabular}

También se consignaron otros factores de vulnerabilidad que se asociaron a la posibilidad de mayor morbilidad de salud mental (Tabla 5). En riesgo vital estuvo casi el $25 \%$ de la muestra. Recibieron agresiones sexuales de más de una persona el $27.5 \%$ de la muestra estudiada. Fueron víctimas de múltiples maltratos el 55\% de las entrevistadas.
Tabla 5. Criterios de gravedad

\begin{tabular}{|l|c|}
\hline Riesgo vital & $7(24 \%)$ \\
\hline $\begin{array}{l}\text { Existencia } \\
\text { de múltiples agresores }\end{array}$ & $8(27.5 \%)$ \\
\hline $\begin{array}{l}\text { Existencia } \\
\text { de múltiples maltratos }\end{array}$ & $16(55 \%)$ \\
\hline
\end{tabular}

Finalmente, la aplicación de la escala de Hamilton a la muestra estudiada, arrojó que un 73\% de la muestra presentaba una depresión leve, un $17 \%$ una depresión moderada y un $10 \%$ una depresión severa.

\section{DISCUSIÓN}

En las mujeres estudiadas, que están cursando una depresión en la vida adulta, la prevalencia de agresiones sexuales en la infancia fue de $27,1 \%$, cifra que se encuentra en el rango establecido por investigaciones restropectivas más amplias. Esto es, cercana al $25 \%$ en población adulta femenina que está cursando un episodio depresivo7.

En términos generales, destaca el alto nivel de traumatización de las mujeres participantes del estudio. Se encuentran agresiones sexuales iniciadas a temprana edad, crónicas y de alta frecuencia. Es importante señalar que tanto la cronicidad como un número de episodios mayor a diez han sido asociados potencialmente a mayor daño psicológico ${ }^{8}$. Estos datos sobre el tipo de agresión sexual y las características principales de éstas (frecuencia, tiempo de duración, edad de inicio) podrían indicar una tendencia al desarrollo de cuadros de morbilidad en salud mental, en donde las agresiones sexuales sufridas en la infancia pueden considerarse como un factor de estrés crucial en el desarrollo psicológico. Aun más, si este estresor perdura durante largos períodos de tiempo, tal como sugieren los datos encontrados, la respuesta de los sistemas biológicos involucrados en la regulación del estrés puede resultar infructífera para estabilizar a la persona, pudiendo producirse una sobrerreacti- 
vidad crónica que puede conllevar consecuencias psicopatológicas en la edad adulta, tal como se aprecia en las mujeres estudiadas.

Los resultados acerca del vínculo con el agresor también van en la línea de lo encontrado en otras investigaciones, esto es, que quienes cometen las agresiones sexuales suelen ser conocidos de la familia y principalmente miembros de ésta. Ello resulta concordante con la mayor tasa de no revelación, en tanto un vínculo cercano induce mayor confusión al momento de revelar o no las agresiones sufridas.

En el grupo de entrevistadas que revelaron lo ocurrido, fue habitual la ausencia de protección de las personas significativas, quienes desestimaron o minimizaron los relatos, lo cual también se traduce concretamente en una nula tasa de denuncias. La denuncia y la credibilidad otorgada a los relatos quedan principalmente asociadas a las agresiones sexuales extrafamiliares.

Conocida es la relación entre la percepción de apoyo posterior a la revelación como factor protector del desarrollo de daño psicológico. Se trataría de un grupo vulnerable, por cuanto es alta la tasa de no revelación y en las que revelaron la sensación de ausencia de apoyo es predominante. Es frecuente encontrar que la percepción de falta de apoyo correlaciona con no develar los hechos, o de haberlo hecho con la retractación de la develación. Por el contrario, la percepción de un patrón intolerante frente a las agresiones y una percepción de apoyo familiar suelen ser factores protectores del riesgo de daño psicológico. En términos concretos, es la ausencia de reconocimiento acerca de los hechos lo que impide la elaboración de aspectos traumáticos, lo cual pudiera posteriormente desarrollarse como síntomas.

Lo anterior se profundiza si coexisten los factores de gravedad descritos. El riesgo vital, la presencia simultánea o subsecuente de más de un agresor y el maltrato de la madre, suelen impactar el pronóstico de aparición de sintomatología en la vida adulta. Se infiere de esto un alto nivel de traumatización en las mujeres estudiadas, lo cual se relaciona con el desarrollo al largo plazo de una vulnerabilidad neurobiológica a trastornos de ansiedad y del ánimo.

Se encontró mayoritariamente personas con episodios depresivos leves, lo cual es coherente con el nivel de complejidad del servicio (atención primaria) en donde se desarrolló la investigación.

Creemos posible señalar que, a la luz de lo expuesto, resulta importante la inclusión en las orientaciones técnicas del Programa Nacional de Depresión ejecutado en el sistema de atención primaria de Chile, de las agresiones sexuales como un factor de riesgo a explorar al momento de diseñar e implementar un tratamiento, de manera que este antecedente sea detectado de manera dirigida.

Desde otro punto de vista, la psicoterapia individual debe contemplarse como posibilidad cuando a la base del trastorno depresivo se encuentra una biografía que incluye trauma9. Se ha demostrado que este tipo de pacientes se beneficia más de una integración de fármacos y psicoterapia, más que la primera por sí solas. Hasta ahora el Plan Nacional de Depresión descrito sólo contempla el uso de fármacos y una intervención psicosocial grupal, a nuestro juicio de corte psicoeducativo, acotada a 6 sesiones.

Queda planteado el desafío de la intervención psicoterapéutica especializada en el ámbito de la atención primaria, lugar privilegiado para dar respuesta a problemas de salud mental y sufrimiento traumático como el descrito.

\section{REFERENCIAS}

1. TRUCCO, M. Estrés y trastornos mentales: aspectos neurobiológicos y psicosociales. Rev Chil de Neuro-Psiquiat 2002; 40 Suplemento 2: $8-19$.

2. VITRIOL, V. Relación entre psicopatología adulta y antecedentes de trauma infantil. Rev Chil Neuro-Psiquiat 2005; 43: 88 - 96.

3. WEISS E, LONGHURST J, MAZURE C. Childhood sexual abuse as a risk for depression in women: psychosocial and neurobiological cor- 
relates. Am J Psychiatry 1999; 6: 816 - 828 .

4. NEMEROFF C, SADEK N. Actualización en neurobiología de la depresión. Rev Psiquiat Uruguay 2000; 3: $462-485$.

5. BARRIENTOS X, FRITSCH R, SOLIS J, ROJAS G. Tratamiento de la Depresión en la Atención Primaria. Rev Psiquiat Clin 2005; 42: 49 59.

6. MINISTERIO DE SALUD DE CHILE, 2001. La depresión: detección, diagnóstico y tratamiento. Guía clínica para la atención primaria.

7. IBACETA, F. Agresión sexual en la infancia y viaje al futuro: clínica y psicoterapia en la edad adulta. Rev Ter Psi 2007, 25: 189 - 198.

8. HUERTA S, MARIC V, NAVARRO C. Factores que intervienen en el impacto del abuso incestuoso sobre la víctima. Rev Ter Psi 2002; 20: $38-46$.

9. JIMENEZ J. El tratamiento psicoterapéutico de la depresión: una visión crítica integrada. Rev Aperturas Psicoanalíticas 2003, disponible en www.aperturas.org, revisado el 10 de enero del 2008 .

Recepción: 12 de mayo de 2008 Aprobación: 13 de noviembre 2008

Usted puede comentar éste y otros artículos publicados en la Revista Chilena de Salud Pública, enviando un correo electrónico a revistasp@med.uchile.cl 